\title{
Review: Bias may contribute to the association between vasectomy and prostate cancer
}

Bernal-Delgado E, Latour-Pérez J, Pradas-Arnal F, Gómez-López LI. The association between yasectomy and prostate cancer: a systematic review of the literature. Fertil Steril. 1998 Aug; 70:191-200.

\section{Question}

In men with a history of vasectomy, is the risk for prostate cancer increased?

\section{Data sources}

Studies were identified in MEDLINE, EMBASE/Excerpta Medica, and IME (Spanish Index Medicus) (1985 to 1996) using terms relating to vasectomy, prostate, prostatic, and cancer. The bibliographies of relevant articles retrieved by the search were reviewed. Searches were also done in Research Activities, published by the U.S. Agency for Health Care Policy and Research and the Spanish network of Research Transfer Offices.

\section{Study selection}

Epidemiological studies were selected if they measured the association between vasectomy and prostate cancer.

\section{Data extraction}

Data were extracted on study design, setting, and period; time during which research was done; sample size; instrument used for gathering exposure and outcome data; effect of measurement units; strength of the association; and statistical methods. Possible sources of bias considered were confounding, selection, detection, nonresponse, regression to the mean, exposure recall, and disease misclassification. The methodological quality was assessed by 2 independent investigators.

\section{Main results}

14 studies were included ( 5 cohort studies and 9 case-control studies). An excess risk for prostate cancer was found in 11 studies (6 studies were statistically significant). The weighted age-adjusted relative risk $(R R)$ for prostate cancer across the 14 studies was $1.23(95 \% \mathrm{CI}$ 1.01 to 1.49 ). The study results varied widely, and the sources of heterogeneity identiffed were type of design, study setting, presence of detection bias, and inadequate selection of controls. The weighted RR for prostate cancer in the cohort studies was 1.13 (Cl 0.84 to 1.52 ) and in the case-control studies was 1,36 (Cl 1.04 to 1.79). The RR for prostate cancer in population-based studies (9 studies) was 1.12 (CI 0.96 to 1.3 ? and in the hospital-based studies ( 5 stud. ies) was 1.98 (CI 1.37 to 2.86). In stud. ies in which detection bias was possibly present, the RR was 1.91 (CI 1.4 to 2.0) in those in which detection bias was less likely, the RR was 1.11 ( $\mathrm{Cr} 0.96$ to $1.2 \%$ ). Studies with adequate selection of controls had an RR of 1.11 (CI 0.94 to 1.31); those with possible selection bias had an RR of 2.24 (CI 1.42 to 3.54).

\section{Conclusions}

Meta-analysis of 5 cohort studies and case-control studies shows an excess rish for the development of prostate cancer in men who have had vasectomy. However, many sources of bias exist among the studies, leading to a probable owerestimation of the association.

Soune of fivding: Fondo de lnvestigations Sanitarias.

For omrespondence: Dr. E. Benal-Delgath Fernando de Antequera $7-1$ D, 5000 Zatugoza, Spain. E-mail apt@onctelprofex

\section{Commentary}

Bernal-Delgado and colleagues have summarised data on the relation between vasectomy and prostate cancer from $14 \mathrm{epi-}$ demiological studies comprising $>200000$ men. The authors made a comprehensive search for ptblished studies (but did not include unpubished work), and they critically evaluated each study for potential bias. They appropriately tabulated the results separately for cohort and case-control studies (because case-control studies often show stronger associations than actually exist) and stratified studies for other potential sources of bias. The most rigorous studies generally showed only a weak association between vasectomy and prostate cancer.

The studies included in this meta analysis investigated the relation between vasec- tomy and the diagnosiz of prostate cancer, not the ocalrence of prostate cancer. Men who have had vasectomies are more likely to seek regular medical care than men who do not and are therefore more likely to have prostate cancer screening (Sackett's "diagnostic access" bias [1]). None of the induded studies attempted to control for medical care-seeking behaviour and are therefore likely to have exaggerated the association between vasectomy and prostate cancer.

Does this metamanalysis exonerate vasectomy? Not necessarily. Given the popularity of vasectomy (approximately one quarter of men 40 to 60 years of age in the United States have had one) and the high prevalence of prostate cancer $>18 \%$ of men in their 60s [2]), even a small increase in the risk for clinically evident disease cond have a large public-health effect.

Future epidemiological studies will grobably not resolve this issue; randomised triab are, of course, not an option. Undersand ing the pathophysiology of prostate cancer may be our best hope for resolving this and other thomy questions about its causes. Peter S. Millarl, MD, PLO Funily Practice Residency Progryts Bangor, Maint, LSA

References

1. Sackett DL. Bias in analytic reseath J Chronic Dis. 1979;32:51-63.

2. Sheldon $\mathrm{CA}$, Williams $\mathrm{RD}$, Fraley Incidental carcinoma of the prostate $x^{\text {te }}$ view of the literature and critical reappras of classification. J Urol. 1980,124:626-3! 